\title{
Comparative Study Between Ovarian Response Prediction Index Versus Anti-Müllerian Hormone, Antral Follicular Count as Predictors of Ovarian Response in Women Undergoing IVF/ICSI Cycles
}

Original Article

\author{
Ahmed M. Maged, Ahmed Amin, Nadeen A. Sherif, Ahmed N. Askalani, \\ Shaza S. SaadAlla
}

Department of Obstetrics and Gynecology, Faculty of Medicine, Cairo University, Cairo

\begin{abstract}
Aim:This study aimed to compare individual predictors as AMH, AFC versus the ovarian response prediction index in determining the ovarian response to controlled ovarian stimulation

Materials and Methods: A prospective cohort study conducted on 84 infertile couples candidate for Intracytoplasmic sperm injection (ICSI) for the first time using either long agonist or antagonist protocol according to clinical evaluation. Participants were classified according to the number of MII oocytes into poor response with 3 or less oocytes and normal responders with 4 or more oocytes.

Results: The study showed that women with poor response were statistically older than those with normal ovarian response (33.1 \pm 5.9 vs. $29.8 \pm 5.4$, respectively). The number of cumulus (12.1 \pm 5.2 vs. $2.5 \pm 1.5)$, MII oocytes (7.8 \pm 3.6 vs. $2 \pm 0.8)$, grade A embryos ( $3 \pm 0.8$ vs. $1.4 \pm 0.9)$ and total number of embryos ( $3.8 \pm 2.2$ vs. $1.7 \pm 0.7)$ were significantly higher in normal responders. ORPI has the highest accuracy in predicting ovarian response (88\%) when compared to $\mathrm{AMH}(83 \%)$ and $\mathrm{AFC}(86 \%)$.

Conclusion: AMH, AFC and ORPI are good predictive of the ovarian response and help in choosing the protocol and gonadotropin dose of induction and prediction of OHSS.
\end{abstract}

Key Words: Antral follicular count (AMH), ovarian response, ovarian response prediction index

Received: 22 September 2019, Accepted: 27 September 2019

Corresponding Author: Ahmed Hussein Saad Mohamed Amin, Department of Obstetrics and Gynecology, Faculty of Medicine, Cairo University, Cairo Tel.: +201005555467, E-mail: ahmed.amin@kasralainy.edu.eg

ISSN: 2090-7265, May 2021, Vol.11, No. 2

\section{INTRODUCTION}

The in vitro fertilization (IVF) process is responsible for about $2-3 \%$ of childbirths in the developed countries ${ }^{[1]}$. It involves many complicated steps ${ }^{[2]}$. To achieve the maximum success, retrieval of adequate number of eggs must be achieved ${ }^{[3]}$.

Ovarian reserve is the size of non-growing primordial follicle population within the ovary. It represents the number of growing follicles their reproductive potential ${ }^{[4]}$.

A poor response to controlled ovarian hyperstimulation is disappointing yet not uncommon in the practice of assisted reproduction technologies ${ }^{[5]}$.

Poor response occurs when the number of oocytes retrieved is insufficient with the resultant higher cancellation rate and lower pregnancy rates ${ }^{[6]}$.
Poor ovarian response occurs in 5 to $35 \%$ of infertile women undergoing controlled ovarian stimulation ${ }^{[7]}$.

Protocols of ovarian stimulation aim to induce development of multiple follicles which eventually increase the number of embryos allowing better selection of transferred embryos according to their grades.

However, large number of follicles produces high non physiological estrogen levels which may have negative effects on the embryo quality and the developing endometrium ${ }^{[8]}$ as the steroid hormones are responsible for endometrial preparation for implantation through cellular, vascular, and immunological effects ${ }^{[9]}$.

To ensure safe and efficient ovarian stimulation, prediction of the ovarian response must be achieved ${ }^{[5]}$. Predicting the ovarian response helps to individualize the ovarian response which results in decrease cancellation 
rate and minimized negative impact of excessive ovarian response $\mathrm{e}^{[8]}$.

The main ovarian response predictors include age, biochemical markers as serum follicle-stimulating hormone (FSH) and anti-Mullerian hormone (AMH) levels and morphological markers as antral follicular count (AFC) ${ }^{[5]}$.

Although oocytes number and quality decrease with age, women with the same age may have different reproductive potentials with different responses to ovarian stimulation. So chronological age may not be predictive as the biological age defined by hormonal and functional markers $^{[10]}$.

The AFC consists of the sum of follicles smaller than 10 $\mathrm{mm}$ detected using transvaginal ultrasound in both ovaries. It is used to predict the ovarian reserve and response to stimulation $^{[11]}$.

The AFC represents the remains of the primordial follicular pool and it is well correlated with chronologic age in fertile women ${ }^{[12]}$. It declines gradually with advancement of age ${ }^{[13]} . \mathrm{AFC}<4$ is predictive of poor ovarian response and high cancellation rates in $\operatorname{IVF}^{[14]}$.

However, the AFC classification limits vary among different researchers. AMH is a member of the beta transforming growth factor family and its solitary source is the granulosa cells surrounding the follicles in their preantral and small antral stages so its level is a direct representative for follicular pool production. AMH is independent of FSH and its level decreases with advancement of age ${ }^{[10]}$.

Both $\mathrm{AMH}$ and $\mathrm{AFC}$ are positively correlated ${ }^{[15]}$. Unlike other serum markers, AMH can be measured at any time in the menstrual cycle ${ }^{[16]}$. However, AMH assay may have different values with improper sample storage and handling techniques ${ }^{[17]}$.

Although ovarian reserve tests are used often, their predictive values are limited, especially in cases of normal values in older women and abnormal values in younger women $^{[11]}$.

None of these individual predictors are considered reliable for either oocytes number and quality or possibility of pregnancy after treatment. The accuracy of individual ovarian reserve tests in prediction of ovarian response is described to be modest in a systematic review ${ }^{[18]}$.

Therefore, the prediction of ovarian response using a single biomarker may not be sufficient for determination of the proper plan of treatment ${ }^{[19]}$.

\section{AIM OF THE WORK}

The aim of this prospective study is to compare individual predictors as $\mathrm{AMH}, \mathrm{AFC}$ versus the ovarian response prediction index (based on combination of $\mathrm{AMH}$, $\mathrm{AFC}$ and age) in determining the ovarian response to controlled ovarian stimulation in women attending for in vitro fertilization (IVF).

\section{PATIENTS AND METHODS}

A prospective cohort study conducted on 84 infertile couples attending IVF unit at Kasr Alainy University Hospital between January 2017 and December 2017. All couples signed an informed written consent after obtaining Kasr Alainy ethical committee approval. Women included in the study were candidate for Intracytoplasmic sperm injection (ICSI) for the first time. Inclusion criteria included women less than 40 years old of age with their basal FSH level less than 10 $\mathrm{mIU} / \mathrm{ml}$. Exclusion criteria included male partners with azoospermia or severely malformed sperms, woman with endocrinological abnormalities (adrenal, thyroid or prolactin level abnormalities), ovarian cysts, abnormal uterine cavity (congenitally abnormal, septate uterus or intracavitary uterine lesions as polypi) detected by hysteroscopy and those with hydrosalpinx (whether unilateral or bilateral).

All participants were subjected to evaluation by history (with special concern to age, type, cause and duration of infertility), examination and transvaginal ultrasound (to calculate AFC (done on days 2-4 of the cycle) and ensure strict inclusion and exclusion criteria)

All participants were subjected to either long agonist or antagonist protocol according to clinical evaluation. The antagonist protocol was offered to women with high liability to develop ovarian hyperstimulation protocol (OHSS) as women with polycystic ovaries. All other women underwent the long agonist protoco ${ }^{[20]}$.

Women in the long protocol received 0.1 mg triptorelin (Decapeptyl; Ferring, Saint-Prex, Switzerland) in the form of daily subcutaneous injection starting from $21^{\text {st }}$ day of the cycle prior to stimulation one till triggering. Gonadotropin induction was started when the following criteria were met : endometrium $<5 \mathrm{~mm}$, serum estradiol $<50 \mu \mathrm{g} / \mathrm{ml}$ and LH level $<5$ IU/L. Induction was started with 150300 IU/ day of human menopausal gonadotropin (Menogon; Ferring, Saint-Prex, Switzerland $)^{[9]}$, then the dose was modified according to serum estradiol and ultrasound monitoring of ovarian response.

Women in the antagonist protocol started gonadotropin induction as in the long agonist protocol starting from cycle day 2.GnRH antagonist cetrorelix (Cetrotide; Merck Serono) was started when more than 1 follicle reached $14 \mathrm{~mm}$, serum estradiol is $\geq 2203 \mathrm{pmol} / \mathrm{L}$ and or serum $\mathrm{LH}$ is $10 \mathrm{IU} / \mathrm{L}^{[21]}$. 
Both Gn and GnRH antagonist were continued till the day of triggering. In both protocols, the women were followed up using transvaginal ultrasonography to measure ovarian number and size, endometrial thickness and pattern starting from $6^{\text {th }}$ day of Gn stimulation daily or every other day according to ovarian response. Triggering was done using 10,000 IU of human chorionic gonadotropin (Pregnyl; Organon, Oss, Netherlands) when $\geq 2$ follicles reached $18 \mathrm{~mm}$ in their mean diameter. The oocyte retrieval was performed through transvaginal aspiration under ultrasonographic guidance 34-36 hours after triggering.

Cycle cancellation was done if less than 3 mature follicles were detected on cycle day 9 or no oocytes were retrieved or patients showed signs of OHSS (E2 $>5000$ $\mathrm{pmol} / \mathrm{L}$, medium sized follicles $>15$ in one ovary). The cycle was cancelled if the transvaginal ultrasonography scan on cycle day 9 indicated inadequate follicular growth ( $<3$ mature follicles), if no oocytes were retrieved on ovum pickup, or if fertilization was unsuccessful ${ }^{[20]}$.

Analysis of oocytes in metaphase II was done followed by ICSI then assessment of fertilization after 16-18 hours and embryo transfer was done on cycle day 3 of ovum pick up using a Labotect semirigid catheter (Labotect, Göttingen, Germany) under guidance of abdominal ultrasound. Daily $100 \mathrm{mg}$ of natural progesterone (Prontogest; Amsa, Rome, Italy) intramuscular injection was done till day of HCG testing done 14 days after ET.

AFC was evaluated during the early follicular phase of a previous cycle before the scheduled treatment. An experienced sonographer, who was blinded to the results of any hormonal assays and the patient's age, performed the evaluation using a conventional 2-dimensional transvaginal ultrasound. The total number of 2-9$\mathrm{mm}$ antral follicles in both ovaries was used for the calculations.

AMH measurement was done through venous blood sample testing before the scheduled treatment (minimum of 30 days) during the early follicular menstrual cycle phase in all women. Enzyme-linked immunosorbent assay (ELISA) kit was used according to the manufacturer's instructions (AMH ELISA; Ansh Labs, Webster, TX, USA). The lowest detection limit of this assay is $0.01 \mathrm{ng} / \mathrm{ml}$.

The ovarian response prediction index (ORPI) values were calculated by multiplying the AMH $(\mathrm{ng} / \mathrm{ml})$ level by the number of antral follicles $(2-9 \mathrm{~mm})$, and the result was divided by the age (years) of the patient. This definition of ORPI is based on previous evaluations that found that the ovarian response to stimulation had positive correlations with the AMH levels and number of antral follicles ${ }^{[22]}$.

ORPI $=($ AMH $\times$ AFC $) /$ Patient age.
The primary outcome parameter was the number of MII oocytes retrieved. Secondary outcomes included the total dose of $\mathrm{Gn}$ used duration of stimulation and clinical pregnancy rate.

Participants were classified according to the number of MII oocytes into poor response with 3 or less oocytes and normal responders with 4 or more oocytes ${ }^{[5]}$.

Power analysis was intended to be done on comparing number of MII oocytes between poor and normal ovarian response. Student's t test for independent samples was chosen to perform the analysis, the $\alpha$-error level was fixed at 0.05 and the sample size was entered to be $12 / 67$ participants for poor and normal ovarian response groups respectively. The intragroup SD was set at the highest recorded one (3.4). Power analysis result showed that the present study is likely to be $>99.9 \%$ powerful in detecting the already recorded difference in number of MII oocytes (5.7 oocytes). Calculations were done using PS Power and Sample Size Calculations Software, version 3.0.11 for MS Windows (William D. Dupont and Walton D. Vanderbilt, USA).

\section{STATISTICAL ANALYSIS:}

Data were described in terms of mean \pm standard deviation or number (percentage) when appropriate. Comparison of numerical variables between the study groups was done using Kruskal Wallis test. Accuracy was represented using the terms sensitivity and specificity. $P$ values less than 0.05 was considered statistically significant. Correlations were tested using the Pearson product-moment correlation. The correlation coefficient (Pearson $r$ ) is interpreted as follows: $<0.2,0.2-0.39,0.4-0.59,0.6-0.7$ and 0.8-1 signify very weak, weak, moderate, strong and very strong, respectively.

Receiver-operating characteristic (ROC) curve analysis was used to examine the value of $\mathrm{AMH}, \mathrm{AFC}$ or ORPI for the prediction of ovarian response. The area under the ROC curves (AUC) was compared using the DeLong method. The AUC is interpreted as follows: $0.9-1.0,0.8-0.89,0.7-0.79$, 0.6-0.69, <0.6 means excellent, good, fair, poor and failed diagnostic or predictive value

All statistical calculations were done using computer program IBM SPSS (Statistical Package for the Social Science; IBM Corp, Armonk, NY, USA) release 22 for Microsoft Windows.

\section{RESULTS}

The results revealed that figure 1 represents flow chart of participants. 
Characteristics of the study population are shown in table 1 . Women with poor response were statistically older than those with normal ovarian response (table1).

There was no significant difference between poor and normal responders regarding male partner age, female BMI, duration, type or cause of infertility (table 1).

Base line hormones named LH, E2, prolactin and TSH showed no significant difference between the 2 groups while baseline FSH was significantly higher in poor responders (table 1). Although the dose of Gn used in poor responders was higher, yet that was not statistically significant (table 1 ).

The induction protocol, duration of stimulation, endometrial thickness at day of triggering, E2 level and progesterone levels measured at triggering day were not significantly different between the 2 study groups (table 1)

The number of cumulus, MII oocytes, grade A embryos and total number of embryos were significantly higher in normal responders (table 1).

The number of embryos transferred and the clinical pregnancy rate were statistically similar in both groups (table 1). The levels of AMH, AFC and ORPI are shown in table1

Diagnostic accuracy tests for predicting poor ovarian response are shown in table 2 and figure 2. The correlation between AMH, AFC and ORPI and stimulation cycle variables are described in table 3 .

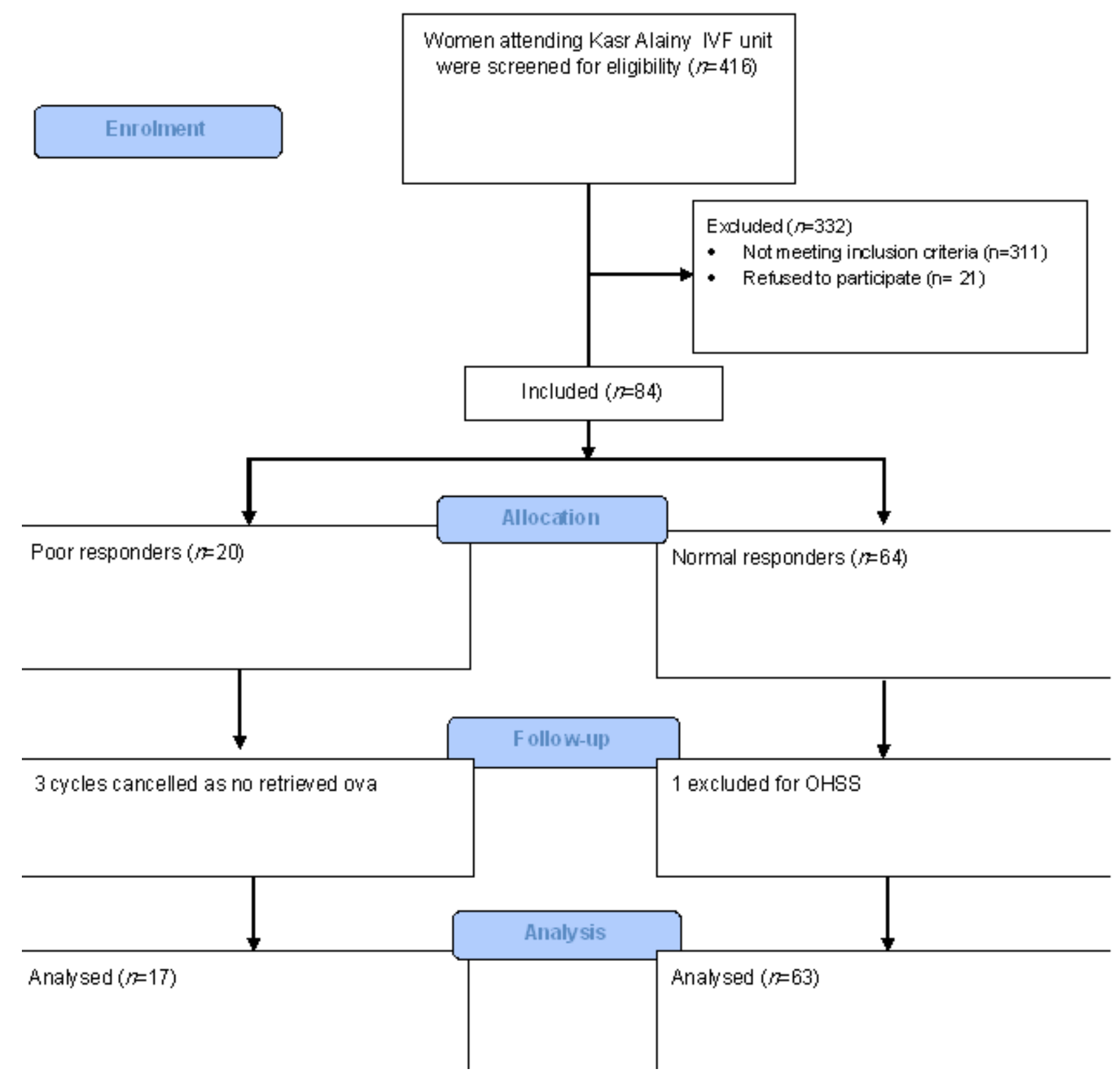

Fig.1: Consort flow chart 


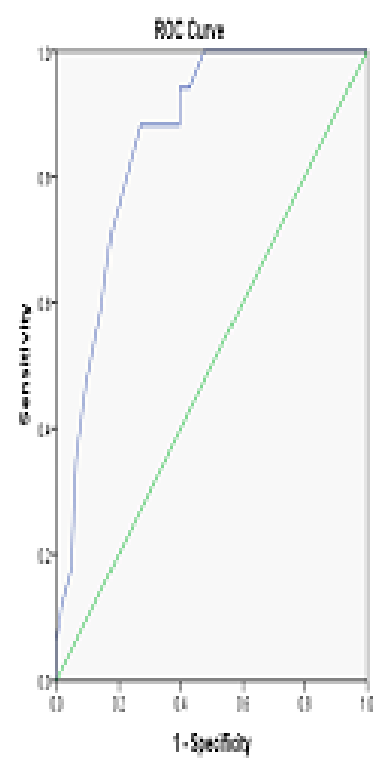

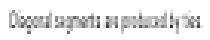

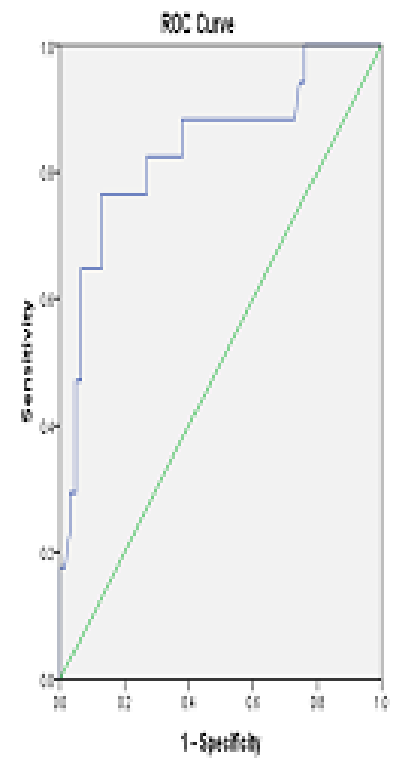

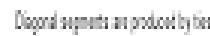

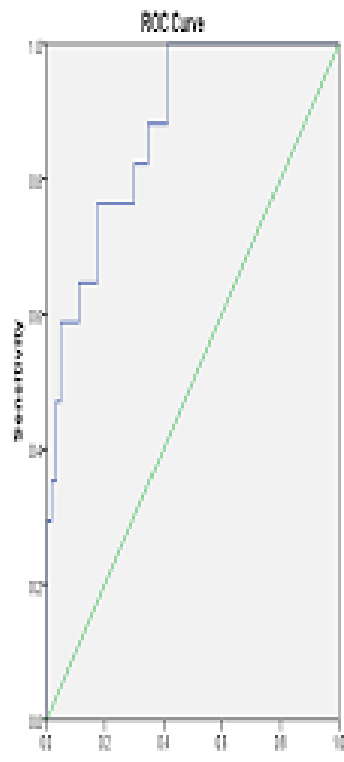

$1 \cdot 5$ ontifion

Fig. 2 : ROC analysis for A:AFC, B: AMH, C: OPRI

Table 1: Characteristics among the two groups

\begin{tabular}{|c|c|c|c|c|}
\hline & & Poor response & Normal response & $\mathrm{P}$ value \\
\hline \multicolumn{2}{|c|}{ Female partner age (years) } & $33.1 \pm 5.9$ & $29.8 \pm 5.4$ & $0.023 *$ \\
\hline \multicolumn{2}{|c|}{ Male partner age (years) } & $37.4 \pm 7.8$ & $34.6 \pm 6.5$ & 0.138 \\
\hline \multicolumn{2}{|l|}{ BMI ( kg/m2) } & $30.6 \pm 5.8$ & $29.8 \pm 6$ & 0.626 \\
\hline \multicolumn{2}{|c|}{ Duration of infertility (years) } & $3.5 \pm 0.7$ & $5.5 \pm 3.6$ & 0.448 \\
\hline \multirow{3}{*}{ Type of infertility } & -Primary & $13(76.4 \%)$ & $57(90.5 \%)$ & \multirow{3}{*}{0.121} \\
\hline & & & & \\
\hline & -Secondary & $4(23.5 \%)$ & $6(9.5 \%)$ & \\
\hline \multirow{5}{*}{ Cause of infertility } & -Male & $9(52.9 \%)$ & $33(52.4 \%)$ & 0.967 \\
\hline & -Tubal & $1(5.9 \%)$ & $12(19 \%)$ & 0.192 \\
\hline & -Ovarian & $4(23.5 \%)$ & $7(11.1 \%)$ & 0.187 \\
\hline & -Unexplained & $3(17.6 \%)$ & $15(23.8 \%)$ & 0.589 \\
\hline & -Others & $1(5.9 \%)$ & $1(5.9 \%)$ & 0.314 \\
\hline \multirow{3}{*}{ Day 3 Hormones } & -FSH (mIU/ml) & $9.1 \pm 5.4$ & $6.2 \pm 2.3$ & $0.001 *$ \\
\hline & -LH (mIU/ml) & $5 \pm 2.4$ & $5 \pm 2.9$ & 0.994 \\
\hline & $-\mathrm{E} 2(\mathrm{pg} / \mathrm{ml})$ & $62.3 \pm 65.5$ & $47.4 \pm 29.9$ & 0.175 \\
\hline
\end{tabular}




\begin{tabular}{|c|c|c|c|c|}
\hline \multirow[t]{2}{*}{ Other Hormones } & -prolactin & $13.4 \pm 5.7$ & $12.2 \pm 5.4$ & 0.444 \\
\hline & $-\mathrm{TSH}$ & $2.9 \pm 2.4$ & $2.1 \pm 1.1$ & 0.176 \\
\hline \multirow[t]{2}{*}{ Induction protocol } & -GnRH antagonist & $8(47.1 \%)$ & $29(46 \%)$ & \multirow[b]{2}{*}{0.94} \\
\hline & -Long agonist & $9(52.9 \%)$ & $34(54 \%)$ & \\
\hline \multicolumn{2}{|l|}{ Gn dose (IU) } & $3555.9 \pm 857.2$ & $3135.2 \pm 932.6$ & 0.098 \\
\hline \multicolumn{2}{|c|}{ Duration of stimulation (days) } & $11 \pm 1.7$ & $12 \pm 2.2$ & 0.095 \\
\hline \multicolumn{2}{|c|}{ Endometrial thickness at triggering $(\mathrm{mm})$} & $9.7 \pm 1$ & $10 \pm 1$ & 0.271 \\
\hline \multicolumn{2}{|c|}{ E2 At HCG triggering (pg/ml) } & $1670.1 \pm 735.3$ & $1965.8 \pm 696.5$ & 0.129 \\
\hline \multicolumn{2}{|c|}{ P4 At HCG triggering $(\mathrm{ng} / \mathrm{ml})$} & $0.6 \pm 0.4$ & $0.7 \pm 0.3$ & 0.297 \\
\hline \multicolumn{2}{|l|}{ No of cumulus } & $2.5 \pm 1.5$ & $12.1 \pm 5.2$ & $<0.001^{*}$ \\
\hline \multicolumn{2}{|l|}{ Number of MII } & $2 \pm 0.8$ & $7.8 \pm 3.6$ & $<0.001^{*}$ \\
\hline \multirow{3}{*}{ Number of Embryos } & Grade A & $1.4 \pm 0.9$ & $3 \pm 0.8$ & $<0.001^{*}$ \\
\hline & Grade B & $0.4 \pm 0.6$ & $0.2 \pm 0.5$ & 0.239 \\
\hline & Total & $1.7 \pm 0.7$ & $3.8 \pm 2.2$ & $<0.001^{*}$ \\
\hline \multicolumn{2}{|l|}{ No of ET } & $1.7 \pm 0.7$ & $2.1 \pm .0 .8$ & 0.051 \\
\hline \multicolumn{2}{|l|}{ Clinical pregnancy } & $6(35.3 \%)$ & $21(33.3 \%)$ & 0.879 \\
\hline \multicolumn{2}{|l|}{$\mathrm{AMH}(\mathrm{ng} / \mathrm{ml}) \#$} & $0.63(1.02)$ & $2.86(2.6)$ & $<0.001^{*}$ \\
\hline \multicolumn{2}{|l|}{$\mathrm{AFC}(\mathrm{n}) \#$} & $6(4)$ & $14(8)$ & $<0.001^{*}$ \\
\hline \multicolumn{2}{|l|}{ ORPI\# } & $0.09(0.46)$ & $1.1(2)$ & $<0.001^{*}$ \\
\hline
\end{tabular}

Data is expressed either as a mean $\pm \mathrm{SD}$ or $\mathrm{n}(\%)$

\#Values are expressed as median and interquartile range

Student t-test was used to reject the null hypothesis

$*=$ Statistically significant.

BMI Body mass index; AMH Antimullerian hormone; FSH Follicle stimulating hormone; LH Luteinizing hormone;E2 Estradiol; GnRH Gonadotropin releasing hormone; Gn Gonadotropin; P4 Progesterone; HCG Human Chorionic Gonadotropin; ET Embryo transfer; ORPI ovarian response prediction index 
Table 2: Diagnostic accuracy tests for predicting poor ovarian response

\begin{tabular}{|c|c|c|c|c|c|c|c|c|c|}
\hline & Cut-off & AUC & Sensitivity & Specificity & PPV & NPV & Accuracy & OR & $\mathrm{P}$ value \\
\hline $\begin{array}{l}\mathrm{AMH}(\mathrm{ng} / \\
\mathrm{ml})\end{array}$ & 0.94 & 0.836 & $70.6 \%$ & $87.3 \%$ & $60 \%$ & $91.7 \%$ & $83 \%$ & 16.5 & $<0.001 *$ \\
\hline $\mathrm{AFC}(\mathrm{n})$ & 10.5 & 0.857 & $94.1 \%$ & $60.3 \%$ & $39 \%$ & $97.4 \%$ & $86 \%$ & 23.4 & $<0.001^{*}$ \\
\hline ORPI & 0.63 & 0.876 & $82.4 \%$ & $69.8 \%$ & $42.4 \%$ & $93.6 \%$ & $88 \%$ & 10.8 & $<0.001 *$ \\
\hline
\end{tabular}

$\mathrm{AUC}=$ area under the curve.

$\mathrm{OR}=$ odds ratio.

$*=$ Statistically significant.

AMH Anti-Mullerian hormone; AFC Antral follicular count; ORPI ovarian response prediction index

Table 3: Correlation between different diagnostic tests for poor response and different cycle variables

\begin{tabular}{|c|c|c|c|c|}
\hline & & $\mathrm{AMH}$ & $\mathrm{AFC}$ & ORPI \\
\hline \multirow[t]{2}{*}{ Dose of Gn } & $\begin{array}{c}\text { Pearson correlation } \\
\text { coefficient. }\end{array}$ & -0.272 & -0.359 & -0.288 \\
\hline & Significance (2 tailed) & $0.015^{*}$ & $0.001 *$ & $0.01 *$ \\
\hline \multirow[t]{2}{*}{ Duration of induction } & $\begin{array}{c}\text { Pearson correlation } \\
\text { coefficient. }\end{array}$ & 0.024 & 0.117 & 0.047 \\
\hline & Significance (2 tailed) & 0.830 & 0.830 & 0.679 \\
\hline \multirow[t]{2}{*}{ Endometrial thickness } & $\begin{array}{c}\text { Pearson correlation } \\
\text { coefficient. }\end{array}$ & $>0.999$ & 0.089 & 0.025 \\
\hline & Significance (2 tailed) & 0.977 & 0.430 & 0.828 \\
\hline \multirow[t]{2}{*}{ MII oocyte number } & $\begin{array}{c}\text { Pearson correlation } \\
\text { coefficient. }\end{array}$ & 0.431 & 0.57 & 0.398 \\
\hline & Significance (2 tailed) & $<0.001 *$ & $<0.001^{*}$ & $<0.001^{*}$ \\
\hline \multirow[t]{2}{*}{ Cumulus number } & $\begin{array}{c}\text { Pearson correlation } \\
\text { coefficient. }\end{array}$ & 0.610 & 0.648 & 0.613 \\
\hline & Significance (2 tailed) & $<0.001^{*}$ & $<0.001 *$ & $<0.001^{*}$ \\
\hline \multirow[t]{2}{*}{ E2 day of trigger } & $\begin{array}{c}\text { Pearson correlation } \\
\text { coefficient. }\end{array}$ & 0.169 & 0.257 & 0.253 \\
\hline & Significance (2 tailed) & 0.133 & $0.021 *$ & $0.024 *$ \\
\hline \multirow[t]{2}{*}{ P4 day of trigger } & $\begin{array}{c}\text { Pearson correlation } \\
\text { coefficient. }\end{array}$ & -0.001 & 0.117 & -0.047 \\
\hline & Significance (2 tailed) & 0.993 & 0.303 & 0.687 \\
\hline
\end{tabular}

*=Statistically significant.

AMH Anti-Mullerian hormone; AFC Antral follicular count; ORPI ovarian response prediction index 


\section{DISCUSSION}

The results found that all the studied markers named AMH, AFC and ORPI are predictive of the ovarian response to stimulation and hence they can help in choosing the protocol of induction, determining the starting dose of gonadotropin and prediction of occurrence of ovarian hyperstimulation.

An array of serum and sonographic tests has been developed to assess ovarian reserve ${ }^{[23]}$. Unfortunately, these tests lack sensitivity and positive predictive value or DOR, particularly if applied to patients at low risk or this process. Identification of the optimal combination of tests and their appropriate interpretation continues to be refined

AMH levels correlate with ovarian primordial follicle number more strongly than do levels of FSH or inhibin ${ }^{[24]}$ and its level decreases before observable changes in FSH or E2 levels. Seifer and colleagues 2011 reported a steady decline in AMH serum levels from $3 \mathrm{ng} / \mathrm{mL}$ at 25 years of age 25 to $1 \mathrm{ng} / \mathrm{mL}$ at the age 35 to $37^{[25]}$.

AFC represents the number of small antral follicles which reflects the size of the resting follicular pool ${ }^{[26]}$. In our study, the female partner age was significantly higher in poor responders.

There is a clear inverse relationship between female age and fertility ${ }^{[27]}$. This loss is primarily attributable to a decrease in oocyte quality and quantity ${ }^{[26]}$.

We found that women in poor responders group had higher FSH than those with normal ovarian response. FSH used to be the simple predictor of ovarian reserve but it had high false negative results (some added simultaneous serum E2 measurement to decrease this fallacy.

With declining ovarian function, the support cells (granulosa cells and luteal cells) secrete less inhibin peptide which is responsible for inhibiting FSH secretion by the anterior pituitary gonadotropes. With loss of luteal inhibin, FSH levels rise in the early follicular phase ${ }^{[26]}$.

According to our findings, poor responders needed higher doses of $\mathrm{Gn}$ than normal responders. Although the difference was not statistically significant, we believe that this difference will be significant if larger sample size was studied.

Our study confirmed that the predictive accuracy of ORPI s higher when compared to AMH or AFC. In our study the dose of Gn was significantly related to AMH,
AFC and ORPI with the highest correlation with AFC followed by ORPI then AMH. The level of E2 at the day of triggering was significantly correlated to AFC and ORPI but not to AMH.

The current study confirmed a highly significant correlation between number of cumulus and MII oocytes and the three studied markers.

Oliveira et al. 2012 evaluated the predictive value of ORPI for ovarian reserve. They found that ORPI has an ideal predictivity for poor ovarian response (AUC: 0.91 ) and a good predictivity for collection of more than 4 MII oocytes (AUC: 0.84) in infertile women. ORPI values of $<0.2$ were described to have the best sensitivity (86\%) and specificity $(89 \%)$ in predicting a poor ovarian responder. These figures were close to our findings which describe a cut off for ORPI $<0.3643$ to predict low ovarian response with a sensitivity of $82.5 \%$ and specificity of $76.5 \%$. They concluded that the use of ORPI can improve the costbenefits of ovarian induction regimens through guiding the drugs type, dose and regimen according to the real patients needs. However, the odds ratios presented by the ORPI were always higher than those presented by all other prognostic factors. But we found that the difference between the AUCs of the 3 predictors (AMH, AFC, ORPI) was not statistically significant (all $p$-values $>0.05)^{[22]}$.

Hendriks et al. 2005 assess the predictive performance of the antral follicle count (AFC) as a test for ovarian reserve in IVF patients and compare this performance with that of basal FSH level. They found that transvaginal ultrasonography is a noninvasive and easy-to-perform method that provides essential predictive information on ovarian responsiveness, like our study which shows that AFC is one of the best predictor for ovarian reserve assessment. We found that AFC less than 8 predict low response with a sensitivity of $73.0 \%$ and specificity of $88.2 \%{ }^{[14]}$.

Muttukrishna et al. 2005, evaluate the relationship between anti-Mullerian hormone (AMH), inhibin $\mathrm{B}$ and antral follicle count (AFC) with ovarian response. They found that inhibin $\mathrm{B}$ had the best positive association with the number of eggs collected and basal AMH is the single best predictor of poor response. AFC has a significant association with the number of eggs collected and is predictive of clinical pregnancy. It is evident that a single parameter is of limited value in predicting ovarian response. But we found no statistical difference between the AUCs of the AMH, and AFC (P-values $>0.05)^{[28]}$.

Lekamge et al. 2007 measured baseline concentrations of serum $\mathrm{AMH}$ and $\mathrm{FSH}$, and $\mathrm{AFC}$ 
from 126 women undergoing IVF treatment. These data were then related to IVF outcomes. As expected, patients with lower serum AMH and AFC produced a significantly lower number of oocytes compared with patients with higher serum AMH/AFC. Fertilization rates in patients with lower serum AMH were significantly inferior compared with patients with higher serum AMH, irrespective of whether IVF or intracytoplasmic sperm injection was used to achieve fertilization. These low AMH patients yielded fewer oocytes, had lower fertilization rates, generated fewer embryos, and had a higher incidence of miscarriage during fresh transfers, ultimately culminating in a halving of the pregnancy rate per IVF cycle compared with the high AMH group.

Lekamge found that the cut off value of AMH less than $1.96 \mathrm{ng} / \mathrm{ml}$ predict low response with a sensitivity of $73 \%$ and specificity $73 \%$; but in our study An AMH level less than $1.02 \mathrm{ng} / \mathrm{ml}$ predict low response with a sensitivity of $87.3 \%$ and specificity $76.5 \%{ }^{[29]}$.

Broer et al. 2009 assess the value of AMH as a test to predict poor ovarian response and pregnancy occurrence after IVF and to compare it with the performance of the AFC. Their study showed that $\mathrm{AMH}$ has at least the same level of accuracy and clinical value for the prediction of poor response as AFC. We also found the difference between $\mathrm{AMH}$ and AFC in predicting the ovarian reserve was not statistically significant (all p-values $>0.05)^{[18]}$.

Younis et al. 2010 tried to find a simple multivariate score that has the potential to predict ovarian reserve, as well as pregnancy rate, in infertile women. One hundred sixty-eight consecutive women underwent their first IVF-ET treatment. Basal ovarian reserve studies, endocrine and sonographic, were performed before starting therapy. After completion of treatment, a logistic regression analysis was performed to examine which parameters significantly determined low ovarian reserve. These parameters were incorporated thereafter in a multivariate score. Logistic regression analysis revealed that age, antral follicle count, basal $\mathrm{FSH}, \mathrm{FSH} / \mathrm{LH}$ ratio, mean ovarian volume, infertility duration, number of previous cycle cancellations, and body mass index were all, in decreasing significance, independent factors that determine low ovarian reserve. The multivariate score was shown to be more accurate in predicting low ovarian reserve than age, day $3 \mathrm{FSH}$, or antral follicle count separately ${ }^{[30]}$.

Islam et al. 2016, compared between AMH and $\mathrm{AFC}$, found that $\mathrm{AMH}$ showed to be a good predictor of ovarian reserve and response. However, AFC compared to other predictors was considered the strongest predictor and there was significant correlation between the response and the quality of oocytes where good responders yielded more oocytes of better quality. Our study agree with the above mentioned study in that both AMH and AFC are both good predictors of ovarian reserve, however we couldn't find statistically significant difference between them (all $p$-values $>0.05)^{[31]}$.

\section{CONCLUSION}

We concluded that the ORPI might be used to improve the cost-benefit ratio of ovarian stimulation regimens by guiding the selection of medications and by tailoring the doses and regimens to the actual needs of patients to achieve optimal response with minimal risks. It may inform patients regarding their reproductive life span and menopausal timing, and also aid in counseling and treatment strategy planning of young female cancer patients receiving gonadotoxic therapy. In addition, it may aid in establishing the diagnosis of PCOS and provide insight into disease severity.

The ideal ovarian reserve test should be convenient, be reproducible, display little if any intracycle and intercycle variability, and demonstrate high specificity to minimize the risk of wrongly diagnosing women as having DOR and accurately identify those at greatest risk of developing OHSS prior to fertility treatment.

Currently, there is no perfect ovarian reserve test, both AFC and AMH level have good predictive value and are superior to day-3 FSH. The convenience of untimed sampling, age-specific values, availability of an automated platform, and potential standardization of AMH assay make this test the preferred biomarker for the evaluation of ovarian reserve in most women. It is important to consider age specific values when interpreting results and to remember that conditions such as PCOS and hormonal suppression can affect the values obtained.

The present study has potential limitations, as despite the proper sample size calculation, we believe that a larger sample size may reveal additional findings as detection of significant difference in $\mathrm{Gn}$ dose, duration of stimulation and pregnancy rates.

We recommend recruitment of a larger sample size in the future studies to be able to reach to the most appropriate protocol for each woman and to reach a significant correlation between ORPI and clinical pregnancy rates.

\section{CONFLICT OF INTEREST}

There are no conflicts of interests. 


\section{REFERENCES}

1. Gearhart J, Coutifaris C. In vitro fertilization, the Nobel Prize, and human embryonic stem cells. Cell Stem Cell. 2011;8:12-5.

2. Boudjenah R, Molina-Gomes D, Torre A, Bergere M, Bailly M, Boitrelle F, et al. Genetic polymorphisms influence the ovarian response to rFSH stimulation in patients undergoing in Vitro fertilization programs with ICSI. PLoS One. 2012;7:1-16

3. Motawi TMK, Rizk SM, Maurice NW, Maged AM, Raslan SAN, Sawaf AH. The role of gene polymorphisms and AMH level in prediction of poor ovarian response in Egyptian women undergoing IVF procedure. J Assist Reprod Genet 2017; 34:1659-1666

4. Practice Committee of the American Society for Reproductive Medicine. Aging and infertility in women. Fertil Steril 2006;86:S248-S252.

5. Maged AM, Nada AN, Abohamila F, Hashem AT, Mostafa WAI, Elzayat AR. Delayed Start Versus Conventional GnRH Antagonist Protocol in Poor Responders Pretreated With Estradiol in Luteal Phase: A Randomized Controlled Trial Reproductive Sciences 2015, Vol. 22(12) 1627-1631

6. Ubaldi F, Vaiarelli A, D’Anna R, Rienza L. Management of Poor Responders in IVF: is there anything new? Bio Med Research International. 2014;2014:1-10.

7. Hendriks DJ, Te Velde ER, Loomann CW, Bancsi LF, Broekmans FJ. Expected poor ovarian response in predicting cumulative pregnancy rates: a powerful tool. Reprod Biomed Online. 2008; 17(5):727-736.

8. Rubio C, Mercader A, Alama P, Lizan C, Rodrigo L, Labarta E, Melo M, Pellicer A, Remohi J: Prospective cohort study in high responder oocyte donorsusing two hormonal stimulation protocols: impact on embryoaneuploidy and development. Hum Reprod 2010, 25:2290-2297

9. Maged AM, Rashwan H, AbdelAziz S, Ramadan W, Mostafa WAI, Metwally AA, Katta M. Randomized controlled trial of the effect of endometrial injury on implantation and clinical pregnancy rates during the first ICSI cycle.Int $\mathbf{J}$ Gynecol Obstet 2018; 140: 211-216

10. Alviggi C, Humaidan P, Ezcurra D: Hormonal, functional and genetic biomarkers in controlled ovarian stimulation: tools for matching patients and protocols. Reprod Biol Endocrinol 2012, 10:9

11. Mira Aubuchon M, Burney RO, Schust DJ and Yao MW. Infertility and Assisted Reproductive Technology. In Berek \& Novak's Gynecology 15th edition. Berek JS (edi.). Lippincott Williams \& Wilkins, A Wolters Kluwer Philadelphia, PA, USA 2012. Chapter 32 pages 1993- 2088

12. Scheffer GJ, Broekmans FJ, Looman CW, et al. The number of antral follicles in normal women with proven fertility is the best reflection of reproductive age. Hum Reprod 2003;18:700-706.

13. Rosen MP, Sternfeld B, Schuh-Huerta SM, et al. Antral follicle count: absence of significant midlife decline. Fertil Steril 2010;94:2182-2185.

14. Hendriks DJ, Mol BW, Bancsi LF, et al. Antral follicle count in the prediction of poor ovarian response and pregnancy after in vitro fertilization: a meta-analysis and comparison with basal follicle-stimulating hormone level. Fertil Steril 2005;83:291-301.

15. Keane K, Cruzat VF, Wagle S, ChaudharyN, Newsholme P, Yovich J. Specific ranges of antiMullerian hormone and antral follicle count correlate to provide a prognostic indicator for IVF outcome. Reprod Biol. 2017;17(1):51-9. doi:10.1016/j.repbio.2016.12.002.

16. Kwee J, Schats R, McDonnell J, et al. Evaluation of anti-mullerian hormone as a test for the prediction of ovarian reserve. Fertil Steril 2008;90:737-743.

17. Broer SL, Broekmans FJ, Laven JS, Fauser BC Anti-Müllerian hormone: ovarian reserve testing and its potential clinical implications. Hum Reprod Update. 2014;20(5):688-701. doi:10.1093/ humupd/dmu020.

18. Broer SL, Mol BW, Hendriks D, Broekmans FJ: The role of antimullerian hormone in prediction of outcome after IVF: comparison with the antral follicle count. Fertil Steril 2009, 91:705-714.

19. Biasoni V, Patriarca A, Dalmasso P, Bertagna A, Manieri C, Benedetto C, Revelli A: Ovarian 
sensitivity index is strongly related to circulating $\mathrm{AMH}$ and may be used to predict ovarian response to exogenous gonadotropins in IVF. Reprod Biol Endocrinol 2011, 9:112

20. The Practice Committee of the American Society for Reproductive Medicine. Ovarian hyperstimulation syndrome. Fertil Steril. 2004;82: 81-86

21. Lainas TG, Petsas GK, Zorzovilis IZ, et al. Initiation of GnRH antagonist on Day 1 of stimulation as compared to the long agonist protocol in PCOS patients. A randomized controlled trial: Effect on hormonal levels and follicular development. Hum Reprod. 2007;22:1540-1546.

22. Oliveira JB, Baruffi RL, Petersen CG, Mauri AL, Nascimento AM, Vagnini L, Ricci J, Cavagna $\mathrm{M}$ and Franco JG. A new ovarian response prediction index: Implications of individualized controlled ovarian stimulation. Reprd Biol Endocrinol 2012 :21;10: 94

23. American Society for Reproductive Medicine: testing and interpreting measures of ovarian reserve: a committee opinion. Fertil Steril 98(6):1407, 2012e

24. Hansen KR, Hodnett GM, Knowlton N, et al: Correlation of ovarian reserve tests with histologically determined primordial ollicle number. Fertil Steril 95(1):170, 2011.

25. Seifer DB, Baker VL, Leader B: Age-specific serum anti-Mullerian hormone values or 17,120 women presenting to fertility centers within the United States. Fertil Steril 95(2074), 2011

26. Thompson MJ, Kho KA. evaluation of the infertile couple. In: Hoffman BL, Schorge $\mathrm{JO}$, Bradshaw KD, et al., editors. Williams Gynecology. $3^{\text {rd }}$ ed: McGraw Hill Education; 2016. p. 427-448 Ch 19 .

27. American Society for Reproductive Medicine: Female age-related fertility decline. Fertil Steril 101(3):633, 2014a

28. Muttukrishna S, McGarrigle H, Wakim R, Khadum I, Ranieri DM, Serhal P:Antral follicle count, anti-mullerian hormone and inhibin $\mathrm{B}$ : predictors of ovarian response in assisted reproductive technology? BJOG 2005, 112:1384-1390.

29. Lekamge DN, Barry M, Kolo M, Lane M, Gilchrist RB, Tremellen KP:Anti-Mullerian hormone as a predictor of IVF outcome. Reprod BiomedOnline 2007, 14:602-610.

30. Younis JS, Jadaon J, Izhaki I, Haddad S, Radin O, Bar-Ami S, Ben-Ami M: A simple multivariate score could predict ovarian reserve, as well as pregnancy rate, in infertile women. Fertil Steril 2010, 94:655-661.

31. Yomna Islam, Mona M. Aboulghar, Alaa ElDin AlEbrashy, Omar Abdel-Aziz. The value of different ovarian reserve tests in the prediction of ovarian response in patients with unexplained infertility Middle East Fertility Society Journal (2016) 21, 69-74 